\title{
Can rotation curves reveal the opacity of spiral galaxies?
}

\author{
A. Misiriotis \\ University of Crete, Physics Department, PO Box 2208, 71003 Heraklion, Crete, Greece \\ e-mail: angmis@physics.uoc.gr \\ Received 16 August 2004 / Accepted 21 April 2005

\begin{abstract}
The observed anti-correlation between the inclination and the slope of the innermost part of the rotation curve can be attributed to dust extinction. However, the implied central face-on opacity of $\tau=2-4$ for the most luminous galaxies is in the optical band. I show that the controversy can be resolved by adopting a model that includes the velocity dispersion of the emitters. Through the use of such a model, I reproduce the correlation between galaxy inclination and the slope of its inner rotation curve without having to resort to excessively high optical depth.
\end{abstract} \\ embarrassingly high compared with studies of edge-on galaxies, which yield central face-on opacities equal to at most $\tau=1$
}

Key words. dust, extinction - galaxies: kinematics and dynamics - galaxies: spiral

\section{Introduction}

Long-slit optical spectra along the major axis of spiral galaxies constitute a main source of information concerning the kinematics of galaxies (see Sofue \& Rubin 2001, for a review). For highly inclined spiral galaxies, however, the long-slit spectrum is distorted by dust, which obscures the innermost parts of the galaxy and eliminates the relevant kinematic information.

As first noted by Goad \& Roberts (1981), a sufficiently optically thick dust disk would yield a solid-body rotation curve regardless of the shape of the actual rotation curve. Therefore, the shape of the rotation curves of edge-on galaxies may be used as a diagnostic for their opacity. This effect was probed by Bosma et al. (1992), who compared the optical rotation curve of NGC 891 with its $21 \mathrm{~cm}$ and CO line kinematics. They concluded that NGC 891 might be moderately opaque at its central regions, but it is definitely transparent at the outer regions. Following this work, Bosma (1995) presented similar observations on a number of edge-on galaxies. His results (under the assumption of an exponential distribution for the dust) imply that the central face-on optical depth of spiral galaxies at $\mathrm{H} \alpha$ is of the order of unity at most.

Along the same lines, Prada et al. (1994) conducted longslit spectroscopy on NGC 2146 at two different wavelengths ( $\mathrm{H} \alpha$ and $[\mathrm{NIII}]$ ) to conclude that while the extinction is significant on the dust lane, it is limited at the outer parts of the disk.

Kregel et al. (2004) studied the stellar kinematics of 17 edge-on galaxies and found that away from the dust lane the rotation curves are far from solid body, thus the effect of extinction is not large.

However, in cases where the long slit spectrum was taken perpendicular to the major axis, one can see the signature of the extinction as a drop at the velocity and the velocity dispersion profile. Kregel \& van der Kruit (2004b) found that in the case of NGC 891, the drop is consistent with a value of the central face-on optical depth at $\mathrm{H} \alpha$ lower than unity, supporting the results of Xilouris et al. (1998), who studied NGC 891 and concluded that its central face-on optical depth is less than one in all visual bands.

Further support for the moderate opacity of spiral galaxies was given by Xilouris et al. (1999), who fitted radiative transfer models on multi-band photometric observations of five edgeon galaxies. The prominent dust lane in these edge-on galaxies enabled them to constrain the amount of dust and to conclude that the implied face-on optical depth in all optical bands is less than unity.

Giovanelli \& Haynes (2002, hereafter GH02) applied the Goad \& Roberts (1981) test in a statistical fashion on a sample of some thousand spiral galaxies and found that there is an anti-correlation between the inclination of spiral galaxies and the slope of the inner part of their $\mathrm{H} \alpha$ rotation curves. This correlation is most prominent in luminosity-class I and II spirals. Valloto \& Giovanelli (2004, hereafter VG04) interpreted this correlation as an effect solely due to dust absorption and argued that the luminosity-class I subsample exhibits an average central face-on optical depth $\tau$ at $\mathrm{H} \alpha$ equal to 2.5, while for the luminosity class II subsample their quoted value was equal to 3.5, significantly larger than other studies have indicated.

In this Paper I attempt to resolve this controversy by introducing a model that reproduces the observed correlation without the need for excessive optical depth. This model takes not only the extinction from dust into account but also the dispersion of the gas velocities in the disk. In Sect. 2 I describe the model. In Sect. 3 I present the long slit spectra of the model and discuss the various factors that affect the derived rotation curve. 
In Sect. 4 I compare my results of the inclination rotation-curve correlation with the observational data. Finally, in Sect. 5 I discuss my results and draw some conclusions.

\section{Model}

The model that I use consists of an axisymmetric exponential disk for the $\mathrm{H} \alpha$ emitters (e.g. Koopmann \& Kenney 2004) whose emissivity is given by

$\eta=\eta_{0} \exp \left(-R / h_{\mathrm{H} \alpha}\right) \exp \left(-|z| / z_{\mathrm{H} \alpha}\right)$

where $\eta_{0}$ is the emissivity normalization constant and is set equal to 1 throughout the rest of the paper. Here $R$ and $z$ are the cylindrical coordinates, $h_{\mathrm{H} \alpha}$ the disk scalelength, and $z_{\mathrm{H} \alpha}$ the disk scaleheight.

The model also consists of an exponential disk for the dust whose extinction coefficient is given by

$\kappa=\kappa_{0} \exp \left(-R / h_{\mathrm{d}}-|z| / z_{\mathrm{d}}\right)$,

where $\kappa_{0}$ is the extinction coefficient at $\mathrm{H} \alpha$ at the center of the disk, $h_{\mathrm{d}}$ is the disk scalelength and $z_{\mathrm{d}}$ is the disk scaleheight. The constant $\kappa_{0}$ is linked to the central face-on optical depth through $\tau=2 \kappa_{0} z_{\mathrm{d}}$.

The mean rotational speed of the emitters in the disk is given by a flat "Polyex" model (GH02), where the rotational velocity $V(R)$ as a function of radius is given by

$V(R)=V_{\max }\left[1-\exp \left(-R / h_{\mathrm{r}}\right)\right]$,

where $V_{\max }$ is the velocity at the flat part of the rotation curve and $h_{\mathrm{r}}$ is the scalelength of the rotation curve. The maximum velocity is taken equal to $100 \mathrm{~km} \mathrm{~s}^{-1}$. Note that the exact value is unimportant and just sets the scale of the velocity.

In addition to the mean rotational velocity, the emitters exhibit random motions described by the radial, the azimuthal, and the vertical velocity dispersions, $\sigma_{R}, \sigma_{\phi}$, and $\sigma_{z}$, respectively. Throughout the rest of the Paper I assume that the velocity dispersion in all directions is $10 \mathrm{~km} \mathrm{~s}^{-1}$, in accordance with the fact that the gas is dynamically cold.

Unfortunately, not only the number of parameters in the above model is quite large, but also the resulting long-slit spectrum (and as a consequence the shape of the rotation curve) depends strongly on each one of them. However, adopting a set of reasonable values can yield a model which describes realistically, if not accurately, an "average" spiral galaxy.

1. Distribution of dust. Recent observations of spirals using SCUBA (Alton et al. 1998a,b), as well as radiative transfer modeling of edge-on galaxies (Xilouris et al. 1999), indicate that the dust follows an exponential distribution with scalelength about equal to 1.4 times the scalelength of the old stellar population and scaleheight about half the scaleheight of the old stellar population leading to a scaleheight to scalelength ratio $q_{\text {dust }}$ around 0.05 . Thus, if in accordance with VG04 we adopt a value of $8 \mathrm{kpc}$ for the scalelength $h_{\mathrm{d}}$ of the dust disk, its scaleheight $z_{\mathrm{d}}$ is $0.4 \mathrm{kpc}$. It must be noted, however, that quoted values for the scaleheight to scalelength ratio of the old stellar population vary from 0.25 (de Grijs 1996; Pohlen 2000) to 0.1 (Xilouris et al. 1999; Kregel et al. 2002), yielding a value of $q_{\text {dust }}$ lying between 0.09 and 0.036 .

2. Distribution of $\mathrm{H} \alpha$ emission. The $\mathrm{H} \alpha$ emission follows star formation in the galaxy, thus making it difficult to use a smooth analytic function to describe its distribution. Generally speaking, its scalelength is larger than the scalelength of the old stellar population, while its scaleheight is smaller. The distribution of $\mathrm{H} \alpha$ emitters is reasonably correlated with the distribution of the dust (Thomas et al. 2004). Thus I assume $h_{\mathrm{d}}=h_{\mathrm{H} \alpha}$ and $z_{\mathrm{d}}=z_{\mathrm{H} \alpha}$.

3. Mean rotational velocity. For the scalelength $h_{\mathrm{r}}$ of the polyex model describing the mean rotational speed of the emitters, I adopt the value of $1.8 \mathrm{kpc}$ found by GH02 from their sample of luminosity-class I galaxies.

\section{Long slit spectra and rotation curves}

The calculation of the simulated long slit spectra is performed in a similar way to Zasov \& Khoperskov (2003) but it is not described here for brevity. Similar calculations of varying sophistication, are discussed by Matthews \& Wood (2001), Baes et al. (2003), Kregel \& van der Kruit (2004b). The rotation curves are derived using the simplest method available, namely the weighted mean of the intensity. While there are better methods that give more reliable results especially for edge-on galaxies (Takamiya \& Sofue 2002), I adopt this method because the data I compare my model with in Sect. 4 were derived using this method.

\subsection{Optically thin case}

In the top panel of the first column of Fig. 1 I show the simulated long-slit spectrum of a model with inclination angle $80^{\circ}$ and no dust. The spectrum follows the the intrinsic rotation curve very well and allows its accurate reproduction, even using the simplest technique of the weighted mean of the spectral line, which I use here. I demonstrate this fact in the middle panel of the first column, where I plot the intrinsic rotation curve (solid line) and the derived rotation curve (dashed line). The small disagreement originates from the fact that the spectral line profile of the simulated long slit spectrum is not symmetrical but exhibits a longer tail towards lower velocities, thus resulting in a somewhat lower value for the mean (see Gentile et al. 2004, for an extended discussion of this subject). This is widely referred to as "projection effect". In the bottom panel I show the major axis profile of the intensity in semilogarithmic scale. Here, the intensity is the integrated intensity along the line of sight.

In the top panel of the second column I plot the long-slit spectrum for an inclination of $85^{\circ}$. The higher inclination increases the projection effect; and as a consequence, the derived velocity curve in the middle panel of the second column differs from the intrinsic. It should be noted, however, that a more sophisticated method would make it possible to retrieve the original rotation curve. In the bottom panel, the value of the intensity is increased compared to the $80^{\circ}$ case, since the 

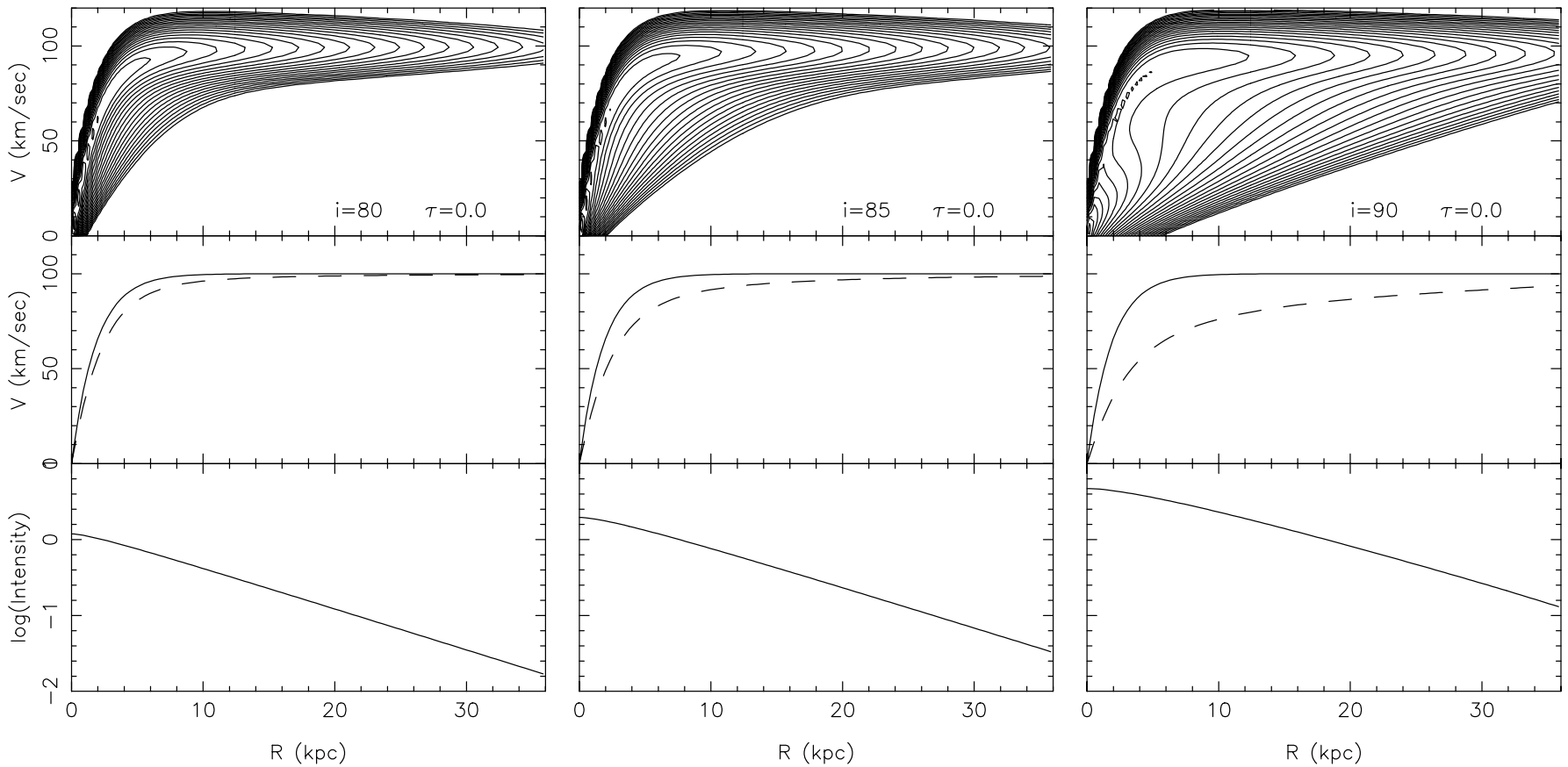

Fig. 1. Long slit spectra of a model without dust. The first column corresponds to inclination $80^{\circ}$, the second column to $85^{\circ}$, and the third column to $90^{\circ}$. The top panels show the long slit spectra. Isocontours are drawn logarithmically so that ten of them correspond to one order of magnitude. The middle panels show the intrinsic (solid line) and the derived (dashed line) rotation curve. The bottom panels shows the intensity along the major axis after taking the inclination of the galaxy into account. Note that as the inclination increases the intensity also increases, as the line of sight intersects with a larger part of the galaxy.

higher inclination causes an increased contribution of the emitters along the line of sight.

In the third column the edge-on case with inclination $90^{\circ}$ is shown. Here the projection effects are severe, causing a significant difference between the intrinsic and the derived rotation curve. Still, the intrinsic rotation curve can be derived accurately from the long slit spectrum through the use of a more sophisticated method (e.g. Takamiya \& Sofue 2002; Kregel \& van der Kruit 2004a).

\subsection{Moderately optically thick case $(\tau=1)$}

In the top panel of the first column of Fig. 2 I show the simulated long-slit spectrum of a model with inclination angle $80^{\circ}$ and central face-on optical depth at $\mathrm{H} \alpha$ equal to 1 . A comparison of this long slit spectrum with the long slit spectrum of the dustless model shows that the effects of the dust are rather limited. In the middle panel I plot the intrinsic (solid line) and the derived (dashed) rotation curve. It is obvious that the small difference is attributed to projection effects rather than to dust extinction. Even in the presence of dust, a sophisticated method would be able to retrieve the intrinsic rotation curve. The effect of the dust is more obvious in the bottom panel where the intensity at the central part of the major axis profile is reduced to about $30 \%$ of its non-extincted value. In the second column, where the inclination is $85^{\circ}$, most of the difference between the intrinsic and the derived rotation curve again originates from projection effects. This, however, does not mean that there is no extinction. In the bottom panel, where the intensity along the major axis is plotted, the absorption is very prominent, as the derived intensity at $R=0$ is reduced to $25 \%$ of its intrinsic value. Finally in the third column, where I show the edge-on case $\left(90^{\circ}\right)$, the effects of the dust become prominent. There the difference of the derived rotation curve from the intrinsic one is due more to dust than to projection effect. Even the use of sophisticated techniques in retrieving the intrinsic rotation curve from the long slit spectrum would fail, as the kinematic information from the innermost parts of the galaxy is absent.

\section{The inclination-rotation curve shape correlation and the opacity of spiral galaxies}

Since the existence of dust and/or velocity dispersion causes the rotation curves of highly inclined galaxies to rise less steeply than in dustless galaxies of the same inclination, the relation between the inclination angle and the slope of the inner rotation curve can be used as a diagnostic for their opacity. A statistical sample of spiral galaxies presented by GH02 shows such a correlation. In a recent paper, VG04 argued that the implied average central face-on optical depth of luminosityclass I galaxies is around 2.5, while for luminosity-class II it is around 3.5 .

The exact amount of dust in luminous spiral galaxies is still uncertain to a factor of 2 (e.g. Alton et al. 2004), their central face-on optical depth in all optical bands, however, seems to be around unity or less (see Xilouris et al. 1999, for a review).

Using the model presented in Sect. 2, I calculate the observed $h_{\mathrm{r}}$ as a function of inclination. In Fig. 3 I show $h_{\mathrm{r}}$ as a function of inclination for four different models exhibiting $\tau=0,0.5,1,1.5$, and 2 . The solid circles show the data 

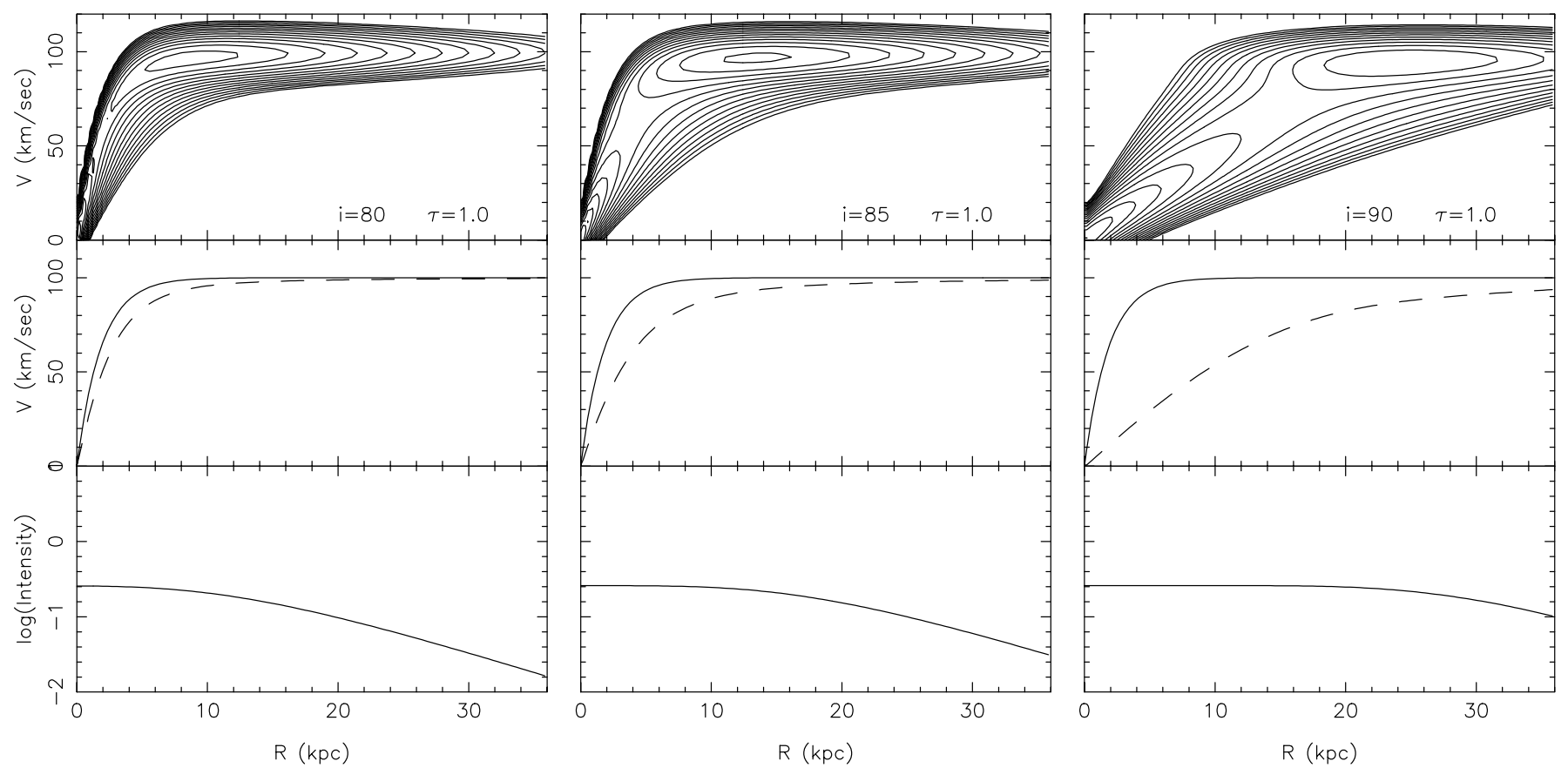

Fig. 2. The same as in Fig. 1 but for central face-on optical depth $\tau=1$.

from VG04. Even for $\tau=0$ there is a significant increase of $h_{\mathrm{r}}$ as the galaxy approaches the edge-on view. As the amount of dust increases, the effects on the rotation curve become more severe and push $h_{\mathrm{r}}$, to higher values. The number of parameters of the model does not allow me to infer the exact central face-on optical depth. Yet a comparison of the model curves with the data allows one to safely state that the central faceon optical depth lies between 1 and 2 . This is about a factor of two lower than the average value of 2.5 derived by VG04 for luminosity-class I galaxies and in accordance with recent studies of the opacity. Note that even for dustless galaxies the projection effects alone are enough to create a significant part of the observed correlation.

\section{Discussion and conclusions}

As already noted, it is nearly impossible to quote an exact value for the central face-on optical depth at $\mathrm{H} \alpha$, due to the fact that the results are sensitive to the following factors:

1. The adopted scaleheight to scalelength ratio $q_{\mathrm{H} \alpha}=q_{\text {dust }}$ of the $\mathrm{H} \alpha$ emitters and the dust, which was assumed equal to 0.05 . As it is also noted by VG04, the results are very sensitive to this ratio.

2. The isotropic velocity dispersion $\sigma_{0}$, for which I have adopted the value of $10 \mathrm{~km} \mathrm{~s}^{-1}$. Had the velocity dispersion been higher, the dustless observed rotation curve would be flatter (see Zasov \& Khoperskov 2003, for an extensive discussion). The additional flattening due to dust would be less and as a result the derived amount of dust would be less.

3. The assumed $\mathrm{H} \alpha$ distribution, especially in the central parts of the galaxy, plays a vital role in determining the magnitude of the effect of dust. In addition to the fact that a very large percentage of spiral galaxies exhibit at least a weak

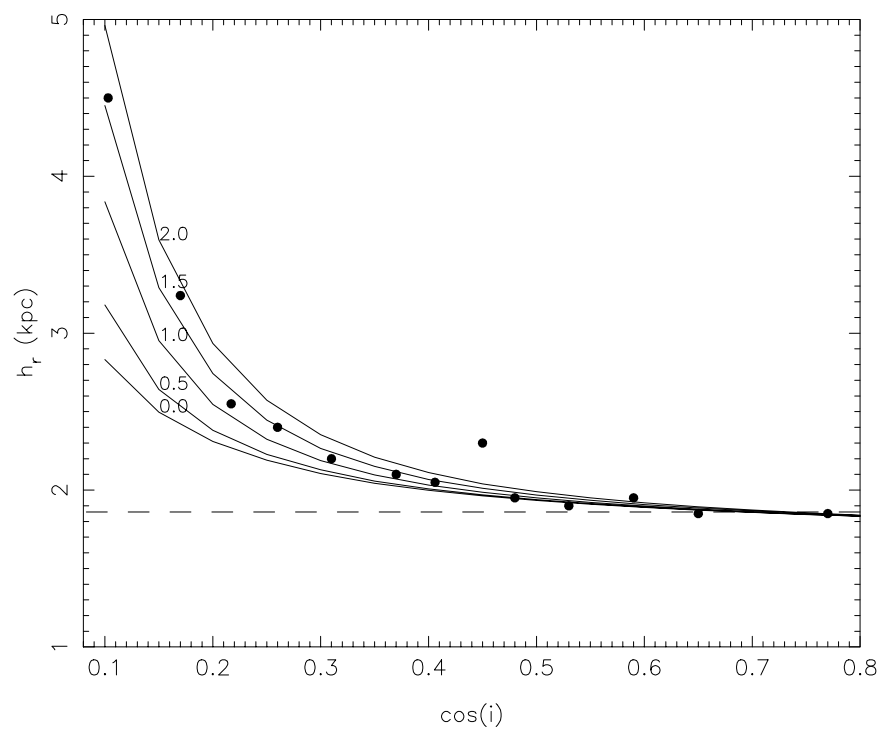

Fig. 3. The observed rotation curve characteristic scale $h_{\mathrm{r}}$ as a function of inclination. The solid circles are data from VG04, while the solid lines show the model results for several different central face-on optical depths. The data points fall roughly between the $\tau=1$ and the $\tau=2$ models. The horizontal line at 1.8 shows the intrinsic value of $h_{\mathrm{r}}$.

bar, the validity of the assumed $\mathrm{H} \alpha$ axisymmetric distribution should be tested. Such a test, however, requires observations and detailed modeling of individual galaxies.

The conclusions of this study can be summarized as follows. Using an exponential model for the spatial distribution of $\mathrm{H} \alpha$ emission and for the distribution of dust in spiral galaxies, I reproduce the $\mathrm{GH} 02$ data that show a correlation between the inclination angle and the slope of the inner rotation curve 
of luminosity class I spiral galaxies. My analysis shows that the average central face-on optical depth at $\mathrm{H} \alpha$ of luminosityclass I galaxies from the GH02 sample is between 1 and 2. This result is in line with the recent study of Xilouris et al. (1999).

While this is not place for a complete review on the recent research on the opacity of spiral galaxies (see Calzetti 2001, for a review of the opacity of star-forming galaxies), it is worth noting that the derived value is a factor of 2 lower than the average value quoted by Misiriotis et al. (2004), who used the spectral energy distribution model of Popescu et al. (2000) to study the dust content of a sample of bright spiral galaxies. Hence, I would like to suggest that detailed studies on individual galaxies using different techniques shall prove indispensable for the refining our ideas on the distribution of dust and the opacity of spiral galaxies.

The comparison of our result with VG04's result shows that neglecting the velocity dispersion from a kinematic model, even when all other assumptions are the same, can significantly overestimate the opacity of spiral galaxies. Thus, I follow Baes et al. (2003), as well as Zasov \& Khoperskov (2003) in stressing the importance of properly including all the factors involved when modeling the kinematic characteristics of edge-on spiral galaxies.

Acknowledgements. This research was sparked by a series of discussions with N. D. Kylafis, whom I would like to thank. I also thank the referee, A. Bosma, for his useful suggestions that improved this paper significantly. This work was supported in part by a Pythagoras II research program of the Ministry of Education.

\section{References}

Alton, P. B., Bianchi, S., Rand, R. J., et al. 1998, ApJ, 507, L125 Alton, P. B., Trewhella, M., Davies, J. I., et al. 1998, A\&A, 335, 807
Alton, P. B., Xilouris, E. M., Misiriotis, A., Dasyra, K. M., \& Dumke, M. 2004, A\&A, 425, 109

Baes, M., Davies, J. I., Dejonghe, H., et al. 2003, MNRAS, 343, 1081

Bosma, A. 1995, in The Opacity of Spiral Disks, ed. J. I. Davies, \& D. Burstein, NATO ASI Ser. C, 469 (Dordrecht: Kluwer), 317

Bosma, A., Byun, Y., Freeman, K. C., \& Athanassoula, E. 1992, ApJ, 400, L21

Calzetti, D. 2001, PASP, 113, 1449

de Grijs, R., \& van der Kruit, P. C. 1996, A\&AS, 117, 19

Gentile, G., Salucci, P., Klein, U., Vergani, D., \& Kalberla, P. 2004, MNRAS, 351, 903

Giovanelli, R., \& Haynes, M. P. 2002, ApJ, 57, 107

Goad, J. W., \& Roberts, M. S. 1981, ApJ, 250, 79

Koopmann, R. A., \& Kenney, J. D. P. 2004, ApJ, 613, 851

Kregel, M., \& van der Kruit, P. C. 2004a, MNRAS, 352, 787

Kregel, M., \& van der Kruit, P. C. 2004b, MNRAS, 355, 143

Kregel, M., van der Kruit, P. C., \& de Grijs, R. 2002, MNRAS, 334, 646

Kregel, M., van der Kruit, P. C., \& Freeman, K. C. 2004, MNRAS, 351,124

Matthews, L. D., \& Wood, K. 2001, ApJ, 548, 150

Misiriotis, A., Papadakis, I. E., Kylafis, N. D., \& Papamastorakis, J. 2004, A\&A, 417, 39

Pohlen, M., Dettmar, R.-J., Liitticke, R., \& Schwarzkopf, U. 2000, A\&AS, 144, 405

Popescu, C. C., Misiriotis, A., Kylafis, N. D., Tuffs, R. J., \& Fischera, J. 2000, A\&A, 362, 138

Prada, F., Beckman, J. E., McKeith, C. D., Castles, J., \& Greve, A. 1994, ApJ, 423, L35

Sofue, Y., \& Rubin, V. 2001, ARA\&A, 39, 137

Takamiya, T., \& Sofue, Y. 2002, ApJ, 576, 15

Thomas, H. C., Alexander, P., Clemens, M. S., et al. 2004, MNRAS, 351,362

Valotto, C., \& Giovanelli, R. 2004, AJ, 128, 115

Xilouris, E. M., Alton, P. B., Davies, J. I., et al. 1998, A\&A, 331, 894

Xilouris, E. M., Byun, Y. I., Kylafis, N. D., Paleologou, E. V., \& Papamastorakis, J. 1999, A\&A, 344, 868

Zasov, A. V., \& Khoperskov, A. V. 2003, AstL, 29, 437 